\title{
ACCELERATORS AND THE ACCELERATOR COMMUNITY ${ }^{1}$
}

\author{
ANDREW SESSLER \\ Lawrence Berkeley National Laboratory \\ Berkeley, CA 94720, U.S.A \\ and \\ ERNEST MALAMUD \\ University of Nevada \\ Reno, NV 89557, U.S.A.
}

\begin{abstract}
In this paper, standing back - looking from afar - and adopting a historical perspective, the field of accelerator science is examined. How it grew, what are the forces that made it what it is, where it is now, and what it is likely to be in the future are the subjects explored. Clearly, a great deal of personal opinion is invoked in this process.
\end{abstract}

\section{Introduction}

It is informative, interesting, fun, and even wise on occasion, to step back and examine our field of accelerator science. This first edition of a review journal is an appropriate time for just such an examination. What are the roots of our science? How did it start? What were the driving forces that made it what it is? How did it evolve? Where is it now? What was the nature of the field, both then and now? Where is it going?

In this short essay we will attempt such an examination. Clearly, a comprehensive job in just a few pages cannot be done, but perhaps, if we look from afar, we can, in broad strokes, paint a picture of our accelerator science. We hope this essay stimulates the reader to think about many different aspects, those raised as well as those ignored. Perhaps these thoughts will lead to a deeper examination of the many technical aspects touched upon, as well as stimulating the reader to reflect on the sociology and communications in the field, and the many diverse sub-fields that promise to become ever more important in the future. This list of sub-fields might include novel methods of acceleration (lasers and plasmas), sophisticated developments in external beam cancer therapy, and applications of accelerators to the energy problem, where accelerators drive power reactors and burn up the longlived components of nuclear waste.

In Part A, we examine the forces that drove this field: nuclear physics, high-energy physics, condensed matter physics, chemistry and biology, national defense, medicine and industry. In Part B, we examine the sociology of the field: the people, the laboratories, and communication and instruction. And, finally, in Part C, we attempt to look into the future.

References have not been added for the sheer number would fill many pages. Rather are presented concepts, laboratories, universities, and the names of some individuals, all well known to the reader.

* This work was supported by the U.S. Department of Energy, Office of Basic Energy Sciences, under Contract No. DE-AC02-05CH11231. 
References, and a different view of the subject of this essay, may be found in the recent book, "Engines of Discovery: A Century of Particle Accelerators," by A. Sessler and E. Wilson, World Scientific (2007).

\section{Part A. Accelerators}

In this part are discussed the fields of science that drove the development of accelerators.

\section{Nuclear Physics}

The development of accelerators began when Ernest Rutherford asked if it were possible to produce nuclear reactions artificially, i.e., not to have to use the energetic products of natural radioactivity. Thus started a race to build machines that would produce particles of sufficient energy to create radioactive isotopes. John Cockcroft and Ernest Walton went the route of electrostatic accelerators, while Ernest O. Lawrence (Fig. 1), stimulated by a paper by Rolf Wideröe and working with his student M. Stanley Livingston, developed the cyclotron. In 1932 when Cockcroft and Walton first artificially produced radioactivity, the cyclotrons at Berkeley had 100 times more intense beams. Yet, because the Californians' detection apparatus was not as well developed, they lost the race to first produce artificial radioactivity.

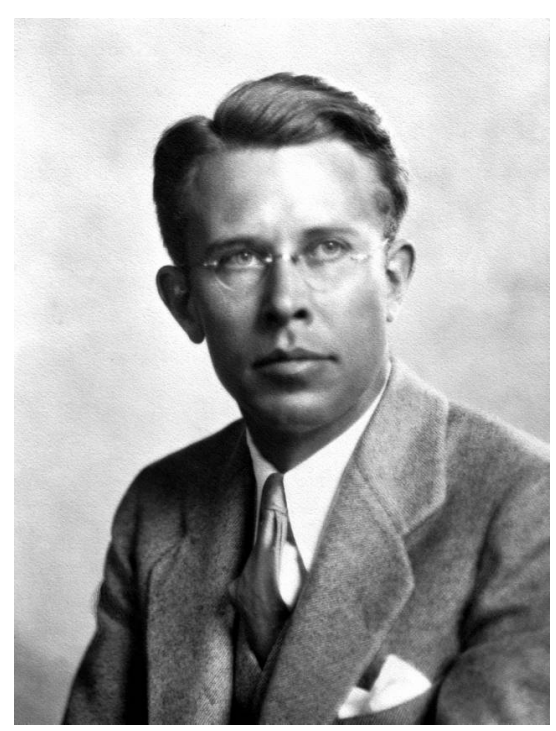

Figure 1. Ernest Orlando Lawrence (1901 1958). Courtesy of Lawrence Berkeley National Laboratory.

Rutherford also inspired R. J. Van de Graaff, an engineer by training, who developed a machine that converted mechanical energy into electrical energy. During the 1930s Lawrence built ever-larger cyclotrons. Just prior to WWII, Donald Kerst, was able to make an electron accelerator, the betatron. and in a subsequent paper with Robert Serber brought sophisticated quantitative mathematical analysis to machines. With these devices nuclear physics flourished in the years prior to WWII.

After WWII, Edwin McMillan and Vladimir Veksler invented phase focusing. With this invention ever more powerful machines could be built for the study of nuclear physics. The 184-inch cyclotron (Fig. 2) produced pions and the field of particle physics in the laboratory, as contrasted with cosmic ray studies, was spawned. At first, particle physicists studied particles interacting with nuclei, and nuclear properties when the bombarding particles were very energetic. Initially those projectiles were protons and later other nuclei, and then various mesons. 


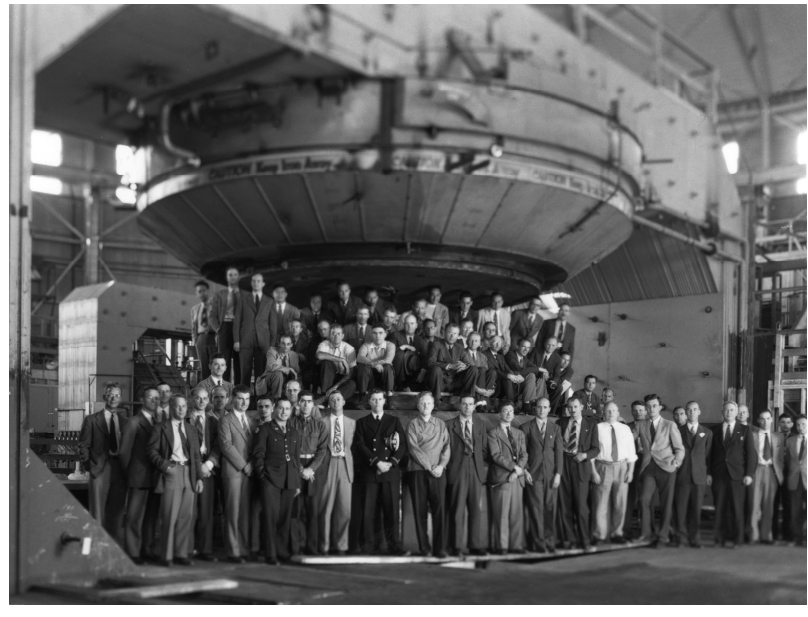

Figure 2. The magnet of the 184-inch cyclotron, the largest that Lawrence built. Courtesy of Lawrence Berkeley National Laboratory.

During this same period nuclear physics drove the development of linear accelerators, first for protons (Luis Alvarez, and then many others) and then electrons (William Hansen, Edward Ginzton, Pief Panofsky and then many others). The culminations of these developments were the proton accelerator LAMPF at Los Alamos and the 2-mile long electron accelerator at SLAC. Nuclear physics also drove the development of spiral sector cyclotrons culminating in TRIUMF. And, of course, it has driven the development of longpulse (even $\mathrm{cw}$ ) facilities for electron-nucleus studies, first at MIT-Bates and then at JLab.

The interest in "discovering" new elements; i.e., elements beyond uranium, spurred activity in Berkeley (12 elements) and also in Dubna (6 elements). More recently GSI has taken up this challenge with considerable, and still on-going, success.

This desire in nuclear physics to study ever more energetic collisions of heavy ions with heavy ions first began with synchrotrons (Bevalac, AGS, SPS), but then eventually led to the RHIC collider. In the future there will be heavy ion collisions at the LHC. The construction and operation of RHIC and the future heavy ion capability at LHC is motivated by the desire to study matter under extreme conditions such as those that are believed to have existed early in the big bang. A possible future development might be the addition of electron-ion collisions to RHIC (eRHIC).

There is interest in nuclear physics, not only in studying matter under extreme conditions-even creating and studying the quark-gluon plasma - but also in studying rare (radioactive) species. To this end, existing facilities, such as those at TRIUMF and Michigan State University have been extended to study radioactive species, while new facilities devoted to this same purpose are being built. A series of nested cyclotrons has been constructed at RIKEN, the FAIR facility is being built at GSI, and in the US the RIA is being designed.

\section{High-Energy Physics}

Simply put, the particle physicists desired ever-higher energy machines while, at the same time having a reaction rate adequate to see ever-smaller cross section events. The accelerator builders provided them with synchrotrons one after the other: the Cosmotron, the Bevatron, CEA, the PrincetonPenn Machine, the Dubna Synchro-phasotron, the AGS, the ZGS, the $70 \mathrm{GeV}$ proton synchrotron near Serpukhov south of Moscow, the KEK Proton Synchrotron, the Fermilab $400 \mathrm{GeV}$ machine and more. The later machines took advantage of the concept of strong focusing (Ernest Courant, M. Stanley Livingston, and Hartland Snyder) as well as the ever-improving technology of vacuum systems, long straight sections, external beams magnets, and rf.

Starting in the 50's, colliders were developed. The driving motivation was HEP and avoiding the square root increase of effective energy in fixed target experiments. Making practical colliders, both hadronhadron, electron-electron, and electronpositron, required producing intense beams ( $\mathrm{rf}$ stacking for hadrons), maintaining the beams 
(an understanding of instabilities and learning how to handle them with non-linearities and feedback), and focusing them in low-beta sections to tiny transverse size (powerful magnets and single-particle beam dynamics.) The first low-beta insertion was done at CEA. These efforts took place in many laboratories, with the original instigators being Donald Kerst, Bruno Touschek, and Gersh Budker (Fig. 3). Experimental work was, at first, at Stanford, CERN, Novosibirsk, Frascati and Orsay. Soon, many colliders were constructed: CEA, SPEAR, ISR, SppS, LEP, PEP I, PEP II, Adone, the VEP series, HERA, the Fermilab Tevatron and, soon to begin operation, the LHC at CERN. The ISR pioneered the science and the technology in ways that no other machine did in the late 60's through the early 70's.

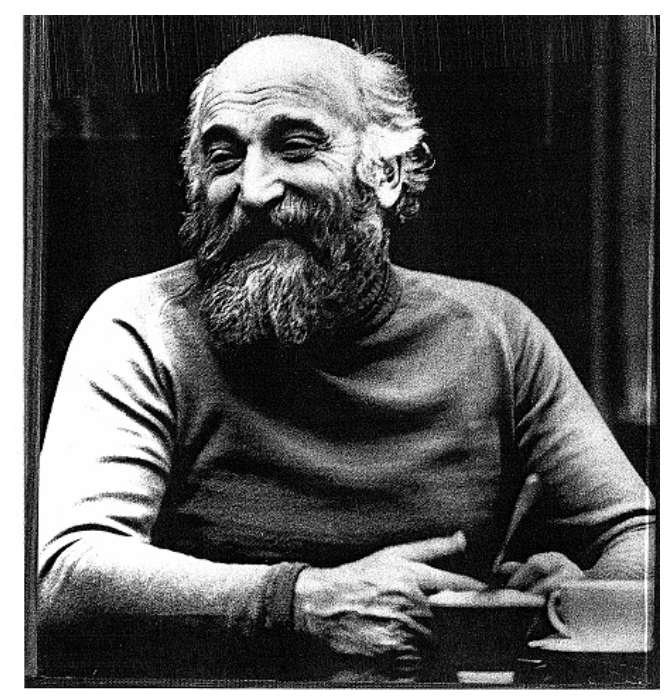

Figure 3. Gersh Budker (1918 - 1977). Courtesy of the Budker Institute for Nuclear Physics.

Robert R. Wilson's vision of the Tevatron, the first superconducting synchrotron was made possible by the ability of Helen Edwards and Alvin Tollestrup. It became the workhorse of the US HEP program, starting as a fixed target machine and then as a proton-antiproton collider in 1986. The Tevatron was a major triumph in accelerator technology and developed most of the technology that made HERA, RHIC and LHC possible. In order to raise luminosity the counter rotating beams were placed on helical orbits, arranged so collisions only occurred at the locations of the large detectors.

The original $400 \mathrm{GeV}$ Main Ring was the first large-scale machine to use a separated function lattice. Another innovation at Fermilab was the construction of the world's first large permanent magnet synchrotron.

Driven by the needs of HEP many accelerator advances were made. Superconductivity became widely used both in rf cavities and in magnets. At the same time, the art of detectors was advanced as new concepts (such as 4-pi detectors, the TPC, silicon detectors) were incorporated into everlarger colliding beam detectors.

Colliders were not only limited to electronpositron and proton-proton colliders. Carlo Rubbia realized that protons and anti-protons could be collided in the SPS. This was accomplished using the concept of stochastic cooling, invented by Simon van der Meer. The SPS converted into a proton - anti-proton collider had sufficient collision energy to produce and discover the $\mathrm{W}$ and $\mathrm{Z}$ bosons. For proton - anti-proton colliders one needs to produce the anti-protons, cool them (stochastic cooling and later electron cooling), and incorporate many other technological advances and inventions that allowed a collider to accumulate particles and store them for a day or longer. All this was, accomplished, first in the SppS collider and later in the Tevatron.

The study of proton-electron collisions was in the initial design of PEP, but support was not forthcoming. The first machine to have ep collisions was HERA in Germany. Looking to the future, circular electron-positron colliders reached their ultimate size in LEP and linear colliders, pioneered by the SLC at SLAC, will be the method of choice in any future machine such as the International Linear Collider (ILC). In 2008, a very large (27 $\mathrm{km}$ in circumference) proton-proton collider, the LHC (Fig. 4), will 
come on line. Improvements to the performance of this machine (a proposed factor of 10 in luminosity), and then the possibility of replacing the guide magnets with more powerful magnets (such as those using $\mathrm{Nb}_{3} \mathrm{Sn}$ superconductor) to increase its energy, will challenge many accelerator scientists in the years to come.

Each of these major facilities required a team of hundreds of skilled and dedicated physicists and engineers, and in each led by an accelerator physicist who was both a good scientist and an accomplished project manager.

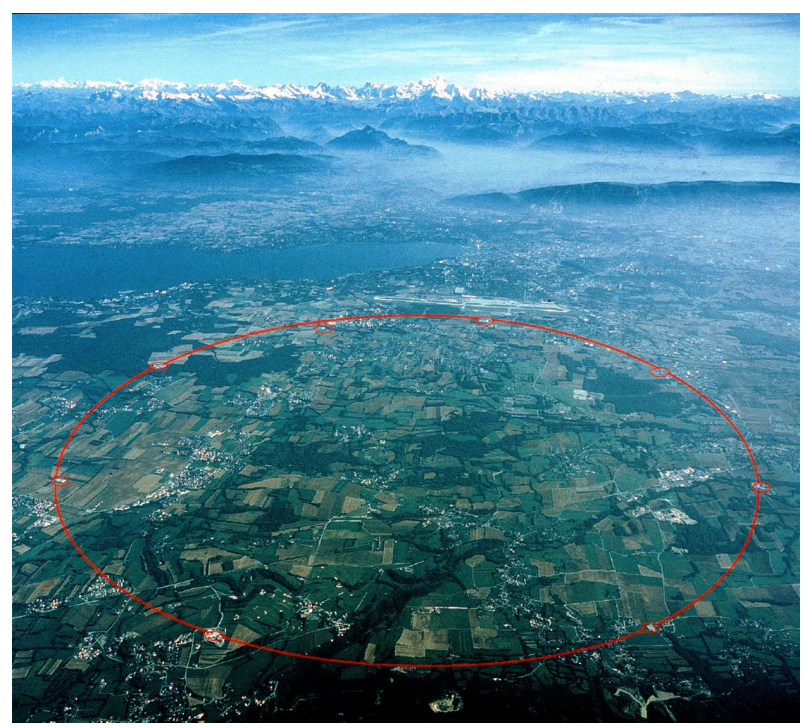

Figure 4. An overview of the European high energy physics laboratory CERN. Superimposed on the photograph is an outline of the ring (deep underground) of the Large Hadron Collider LHC. Courtesy of CERN.

Important science has emerged from the non-accelerator work at the Kamioka detector, the Super K detector and the SNO detector: the experimental demonstration that neutrino oscillations were the source of the solar neutrino problem. Detailed studies of neutrino oscillations and neutrino mass are being investigated by the development of major accelerator-based neutrino experiments. The first was $\mathrm{K} 2 \mathrm{~K}$ at KEK, and Super-K at KEK, and at Fermilab both the MINOS and MiniBooNE experiments, and, most recently, by CERN to Gran Sasso. At J-PARC a very intense neutrino beam will be created and sent to Kamiokande. Neutrino beams will play a very important role in the future.

The first accelerator neutrino experiments were proposed independently by Mel Schwartz and Bruno Pontecorvo around 1958 when they realized that the pion beams that would be produced by the AGS and the CERN PS, then under construction, could be used to produce reasonably intense neutrino beams. Neutrino experiments were a central part of the fixed target programs at the Fermilab Main Ring, its successor the Tevatron and the CERN SPS.

With the work on the various accelerators listed, and much theoretical work, first weak interactions were understood and, then, subsequently the standard model was established. Thus accelerators were at the heart of one of the -- perhaps the - greatest accomplishment of physics in the last half of the $20^{\text {th }}$ century.

\section{Condensed Matter Physics, Chemistry and Biology}

During the last few decades the scientific disciplines playing a very large-perhaps the largest - role in accelerator development have not been nuclear or high-energy physics, but rather condensed matter physics, chemistry and biology. Sure, there has been LEP, LHC, RHIC, the nested cyclotrons at RIKEN, and FAIR, but the major recent development has been in synchrotron radiation sources (Fig. 5) and, most recently, in spallation neutron sources. 


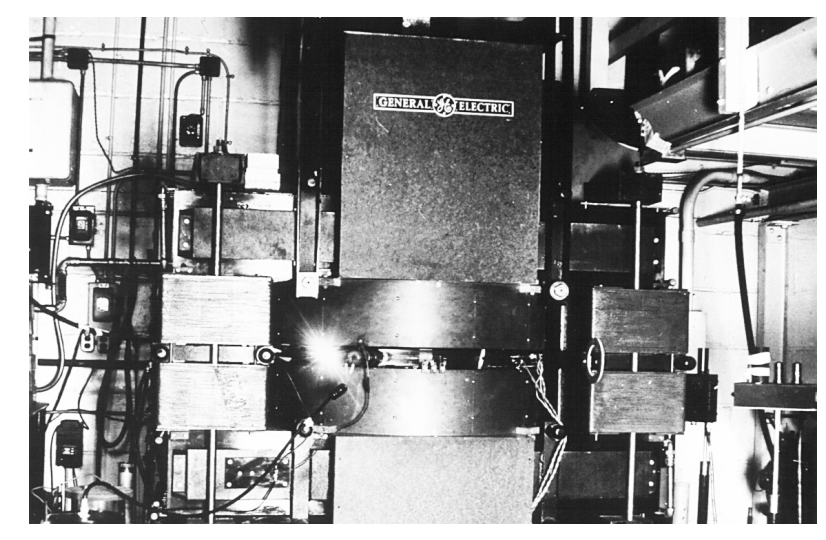

Figure 5. The first observation of synchrotron radiation was made from this $300-\mathrm{MeV}$ electron synchrotron at the General Electric Co. at Schenectady, built in the 1940's. Courtesy of the U.S. Government.

These machines that produce intense beams of synchrotron radiation or neutrons are used in condensed matter physics for the study of the magnetic properties of matter (such as is used in computer hard drives), the origin of high temperature superconductivity, wear in turbines, the catalysis in industrial chemical processes and many other studies. In chemistry the use of synchrotron radiation has been used to study the structure of molecules, and now is allowing the study of the dynamics of chemical reactions. Some have said the last few hundred years of chemistry was devoted to the study of equilibrium states, while the next few hundred will be devoted to dynamical studies.

In biology, and medicine, synchrotron radiation is used to elucidate protein structure and cell structure. A very large activity is in the design of pharmaceuticals. Synchrotron radiation is also employed in environmental sciences, geosciences, and even in art and archeology.

Most often neutron spallation studies are combined with synchrotron X-ray studies, for the two are complimentary in their sensitivity as a function of atomic number.

The idea of using a storage ring to produce synchrotron radiation came from the MURA
Group. The very first such rings were TANTALUS in Madison and the ring at NIST. In the early 70's some rings developed and operated for high-energy physics were used as synchrotron radiation sources. In the US, the CEA was turned off for high-energy physics. SPEAR, after several years of parasitic operation, became a dedicated synchrotron user facility. It was appreciated that storage rings with insertion devices, undulators and wigglers, in long straight sections gave far superior X-ray beams. The first permanent magnet wiggler was built in Berkeley and employed at SPEAR. These machines were called Second Generation, in contrast with the First Generation that simply used bending magnets to generate $\mathrm{X}$-rays. The use of these $\mathrm{X}$-ray sources became so valuable that "user demand" resulted in something like 70 of them having been built in many different countries.

The next step in this evolution was the construction of very energetic rings specially designed for their resulting X-ray beams. This Third Generation includes the $6 \mathrm{GeV}$ ESR in Grenoble, the $7 \mathrm{GeV}$ APS at Argonne, and SPring-8 $(8 \mathrm{GeV})$ in Japan, as well as the lower energy (but intense) ALS in Berkeley. At present the NSLS at Brookhaven is being augmented with a whole new facility in the $800 \mathrm{M} \$$ range. And a number of lower energy (a few GeV) Third Generation facilities are now being constructed in various places around the world.

The free electron laser (FEL) has been under development since it was invented and demonstrated by John Madey et al, in the late 70 's. Since that time the radiation wavelength has become shorter and shorter, the latest being the FLASH facility at DESY operating in the VUV. A major Fourth Generation machine, built with a linac (1/3 of the two-mile linac) and an FEL is under construction at SLAC. This device, called the LCLS, will produce coherent radiation of 1.5 Angstroms $(0.15 \mathrm{~nm})$. A similar device is under construction at SPring- 8 and an XFEL using superconducting RF is under construction at DESY. Other groups are considering 
recirculating linac FELs, while some groups (like LBNL) are considering whole complexes of FELs. The aim is to produce many pulses per second $(\mathrm{MHz}$ as contrasted with $100 \mathrm{~Hz}$ at SLAC) and beams of very short temporal extent (100 attoseconds). The requirements on emittance, timing, stability and pulse manipulation are challenging indeed.

While X-ray sources have undergone the development described above, neutron sources - for many of the same applications as X-ray beams - have also undergone major development. The SNS in Oak Ridge has been the major accelerator project in the USA for the last decade (just as LCLS is for this decade). The superconducting linac is the highest energy sc hadron linac ever built, while the storage ring was a major challenge. A similar facility is being built in Japan ( $\mathrm{J}$ PARC) and plans are under way to build spallation facilities in Europe and China. The development of pulsed neutron sources also has an interesting history involving contributions from many laboratories and people. The first one was built at Argonne using the ring components of the old 1.2 GEV Cornell machine. Its successor IPNS provided the best and most reliable source of pulsed neutrons for the US spallation neutron source community for many years. After NIMROD was turned off around 1977 ISIS was built at Rutherford. For the past 25 years it has been the most productive neutron spallation source in the World, only now is SNS overtaking it. Ultimately LANSCE (LAMPF renamed), the pulsed storage ring (PSR), and the Lujan center overtook the Argonne IPNS in the US around 2004. The SNS at Oak Ridge was started in 2000. These earlier machines pioneered the beam and instrumentation technology.

\section{National Defense}

The first use of accelerators in national defense was when Lawrence used the not-yetcompleted 184-inch cyclotron to separate $\mathrm{U}^{235}$ from $\mathrm{U}^{238}$. This then resulted in the construction of over a thousand Calutrons through which passed all of the material of the Hiroshima bomb. These were more like spectrometers than accelerators, but the operation involved dealing with space charge effects very similar to those in accelerators.

During World War II, the Germans, the British and the Americans employed betatrons. The Germans hoped to be able to blind the pilots of Allied bombers, the British used them to X-ray unexploded bombs and, therefore, help in the defusing process, and the Americans employed a betatron at Los Alamos to help in the development of the atomic bomb. It was one of these British betatrons that, after WWII, was converted into one of the very first synchrotrons.

Shortly after WWII, the American AEC became concerned that there was not enough readily available uranium to allow the buildup of what would become a very large nuclear weapon inventory. Thus the Material Testing Facility (MTA), which was to be essentially a spallation source of neutrons, was authorized. This machine was a very large linear accelerator (the accelerating tank was 90 feet long and 30 feet in radius) that was promoted, designed, and built by Lawrence and coworkers, but in fact, never worked.

The desire to protect the American nation from incoming missiles was responsible for the development of induction accelerators.

Long before the SDA proposal ("Star Wars") a conference was held in 1952 at which particle beam weapons were discussed. The actual SDA program, whose purpose was missile defense, was initiated in 1958, under which the Experimental Test Accelerator (ETA) and the Advanced Test Accelerator (ATA), (both linear induction machines) were constructed at Livermore (Fig. 6). During "Star Wars" times these machines were used to develop ground based free-electron lasers (FELs). These ideas never resulted in antimissile weapons. 


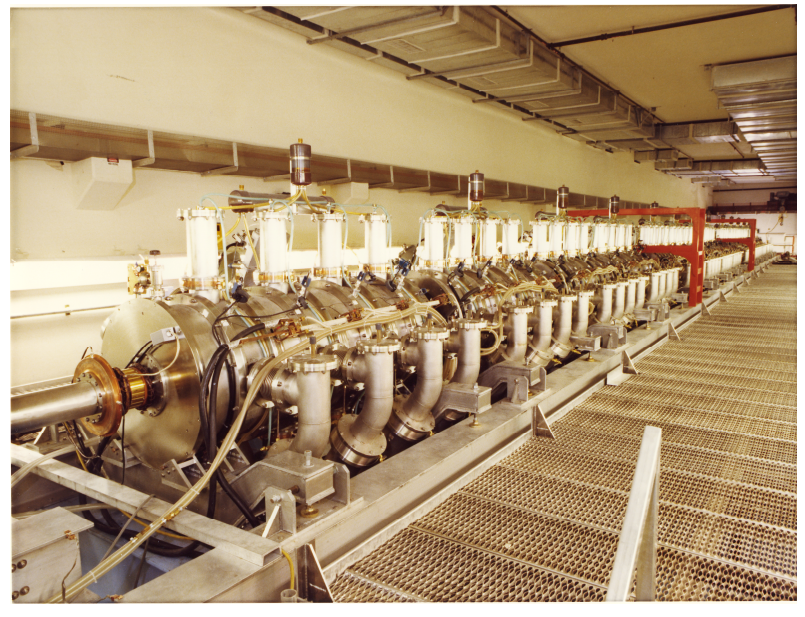

Figure 6. Induction linac FXR at Lawrence Livermore National Laboratory, completed in 1982 to study the implosion process in nuclear weapons. Courtesy of Lawrence Livermore National Laboratory.

Induction accelerators have been effective in producing X-ray pulses to study the hydrodynamics of nuclear weapon implosions. The FXR was built at Livermore and, just being commissioned, is the DARHT Facility at Los Alamos.

During the period when SDA concepts were being explored, Los Alamos built, and then operated, an accelerator in space. This project was called BEAR, a Beam Experiment Aboard a Rocket. More importantly, WNRF, the Weapons Neutron Research Facility storage ring system at Los Alamos (operates in two different pulsed modes using protons from LAMPF) was successful in its mission and has led to the discovery of the electron cloud instability, a matter of importance in many machines.

The US Navy has an intense interest in defending its large ships. One possibility is an on-board FEL and in this regard it has supported the development of ever-higher average power FELs. This activity has not yet resulted in an anti-cruise missile, or an antishell weapon, but the necessary power to achieve that end is within sight.

\section{Medicine}

John Lawrence, a medical doctor and brother of Ernest Lawrence, working in Berkeley with the cyclotrons, started the field of nuclear medicine in 1936. Subsequently there has been a large need to produce radioactive nuclei both for diagnostic purposes (PET) and for cancer therapy purposes (an example is the treatment of thyroid tumors). This has driven the development of small cyclotrons $(18 \mathrm{MeV}$, $40 \mathrm{MeV}$, etc.). These machines are robust, reliable and relatively inexpensive and are usually located in major city hospitals. One large commercial firm has produced over 1,000 of them.

The medical advantage of high energy Xrays as an external beam for cancer therapy has driven the development of X-ray producing linacs. At first these were rather large spatially fixed machines operating below the energy for producing radioactivity, i.e., below about 10 $\mathrm{MeV}$. Now there are compact linacs (still operating below $10 \mathrm{MeV}$ ), which are reliable and mounted directly on a gantry, so they may be rotated about the patient. A great many of these machines are in hospitals around the world. One large commercial supplier is manufacturing two or three such machines every day! .

It has been appreciated, in the medical community, that external hadron beam therapy for certain types of cancer is advantageous. This realization has driven - and right now is intensively driving - the development of a variety of machines. They include cyclotrons and synchrotrons, with cyclotron-fed linacs, and Non-Scaling Fixed Field Alternate Gradient (NS-FFAG) accelerators on the horizon. Also, a good number of aged facilities, often no longer supported for nuclear physics, are being converted to use for medical applications (examples are in Indiana and Catania.)

\section{Industry}

Industry is a prime user of accelerators. The market, consisting of constructing accelerators, 
with their associated peripheral equipment, is estimated to be more than $1000 \mathrm{M} \$$ /year. Unlike the disciplines described above, industrial use of accelerators has not led to the development of new types of accelerators or even to the many developments of accelerator technology that has allowed advances. However, industrial use has stimulated nontrivial, ever more sophisticated forms of "conventional machines" and, of course, has depended on the community of accelerator scientists that are needed to make this happen. We describe present use below, but in the future one can expect that industrial use of accelerators will become even larger.

The primary use of accelerators is in the semiconductor industry where doping silicon with Boron or Phosphorus (forming $\mathrm{p}$ - or $\mathrm{n}$ junctions) requires a range of energies from $100 \mathrm{keV}$ to $1.5 \mathrm{MeV}$. In the high-energy portion of this range, linacs and tandem Van de Graaffs are used; electrostatic machines cover the mid-energy range (tens of $\mathrm{keV}$ ) and the low-energy range (hundreds of $\mathrm{eV}$ ). These machines are commercially produced and sold primarily in the US, Europe, Malaysia, Singapore, China, South Korea, Japan, and Taiwan.

The wafers used in satellites (SOI, Silicon on Insulator), and also in HEP detectors, are radiation hard. The insulating layer of oxide is deposited with oxygen ion beams in the 100 $\mathrm{keV}$ range. These types of wafers are used by companies such as IBM and Honeywell in the US to manufacture special types of chips. A new technique "Smart Cut" which requires energetic hydrogen ion beams is now being employed for the manufacture of SOI wafers.

At present, chips are made with photoresist and masks and VUV light from a $\mathrm{KrF}$ laser. In order to make ever-smaller objects a different lithography technology is required and that may well be the use of electron or ion beams, probably in the range of $70 \mathrm{keV}$ and produced electrostaticly.

Turning from semiconductors to other types of materials we find a wide use of accelerators in machining, cutting, surface modification and surface analysis. For example, there is Secondary Ion Mass Spectroscopy (SIMS) that requires a tightly focused ion beam. Currently a number of Japanese companies are producing tens of electrostatic machines per year. These give 35 $\mathrm{keV}$ ions and a spot size diameter of $2 \mathrm{~nm}$.

Another use of accelerators is in the sterilization of spices, food, medical instrumentation, and even the US mail addressed to sensitive places (White House, Pentagon, Congress, etc.). X-rays, or even direct electron beams are employed in these systems. Most of these applications require very high power machines, typically electrons at $6 \mathrm{MeV}$. These are either voltage multiplying devices (Novosibirsk) or microtron-like devices called Rhodotrons (manufactured by IBA). Tens of units are being produced each year.

There are many other industrial applications of accelerators. In common use is the enhancing of the brilliance of gemstones. A special application is in the treatment of the sugar being transformed into ethanol (such as in Brazil). Subjecting the sugar solution to 1.5 $\mathrm{MeV}$, electrostaticly generated electrons, kills bacteria in the solution and increases the conversion efficiency by $4-5 \%$. Accelerators make X-rays for studying the continued integrity of airplane wings, bridges, and other structures. For this application, at least one company has designed a compact, portable, betatron that it is producing and selling commercially.

\section{Part B. The Accelerator Community}

In this Part we touch upon the community of accelerator scientists. It is interesting to see how the community developed, how the laboratories within which many of them worked and, finally, how these scientists communicate and interact with each other.

\section{The People}

In the early 1930s, there were no accelerator scientists. Accelerators were designed 
(minimally) and built by physicists/engineers who "moved over" from other branches of physics. For example, Cockcroft was an atomic physicist, Odd Dahl an oceanographer, Van de Graaff a mechanical engineer, Lawrence a cosmic ray specialist, McMillan mostly a chemist but also a nuclear physicist, Alvarez a nuclear physicist, Hansen a physicist interested in electromagnetism, Ginzton an electrical engineer (and also a physicist), and Panofsky a nuclear physicist.

Wideröe's proposal for resonant acceleration in a linac like device preceded Lawrence's principle for a cyclotron and influenced Lawrence. Lawrence and Livingston built the first operating cyclotron that spawned a long line of cyclotrons and then later synchro-cyclotrons and synchrotrons. Lawrence's cyclotrons created big science and extraordinary science was done with them. John Adams, built two machines (the PS and SPS) and provided the vision for the VBA presented at a meeting in New Orleans in 1975. The LHC is the incarnation of the VBA. Burton Richter and others developed collider technology with the Stanford electron collider. SPEAR reshaped particle physics and helped to shape synchrotron light sources. Richter was also the force behind LEP and the SLC.

At Cornell, before becoming the Founding Director of Fermilab, Robert Wilson (Fig. 7) built a series of electron synchrotrons and fostered a whole school of accelerator builders from among his colleagues and students. The first person to seriously propose electron-

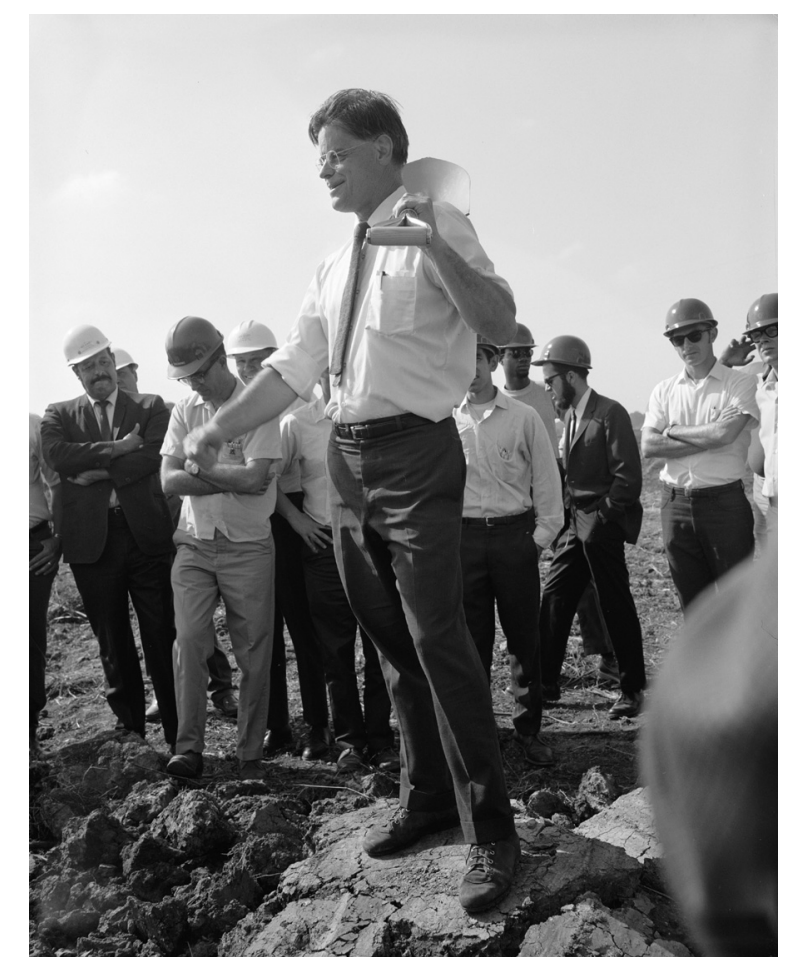

proton collisions was Bjorn Wiik for DORIS, important not only for physics but accelerator science.

Figure 7. Robert Wilson breaks ground October 3, 1969 for the $200 \mathrm{GeV}$ proton accelerator, which came into operation in 1972. Courtesy Fermilab.

Although much activity in the field consists of building and improving accelerators following well established and proven ideas, nevertheless new innovations with great promise are frequently proposed. John Dawson and Toshi Tajima were the first to call attention to the possibility of using plasmas and lasers for particle acceleration. Robert Palmer at Brookhaven has been spearheading the activity, and making important contributions, to neutrino factories and muon colliders. Nicholas Christofilos, while working at Livermore, invented induction accelerators as part of the fusion energy program.

The very first training of students for a $\mathrm{PhD}$ in accelerator physics was by E.O. Lawrence. Amongst his early students were M. Stanley Livingston ("Lawrence was my teacher when I built the first cyclotron - he got a Nobel Prize for it - I got a PhD."), David Sloan, and later Jackson Laslett. Wideröe (Fig. 8) was self-motivated, but he was very much the exception. After WWII, many students were trained, in many different countries, to obtain a $\mathrm{PhD}$, in accelerator science. It is impossible to create a comprehensive list of Ph.D. supervisors who having been active in training students, but a few institutions in which they have taught come to mind. They include, just to name a few in the United States, the University of California in Berkeley, UCLA, Caltech, the University of Chicago, Cornell University, the University of Illinois, Indiana University, the University of Southern California, Stanford, New York University at Stony Brook, the University of Maryland, MIT, Princeton, and the University of Wisconsin. 


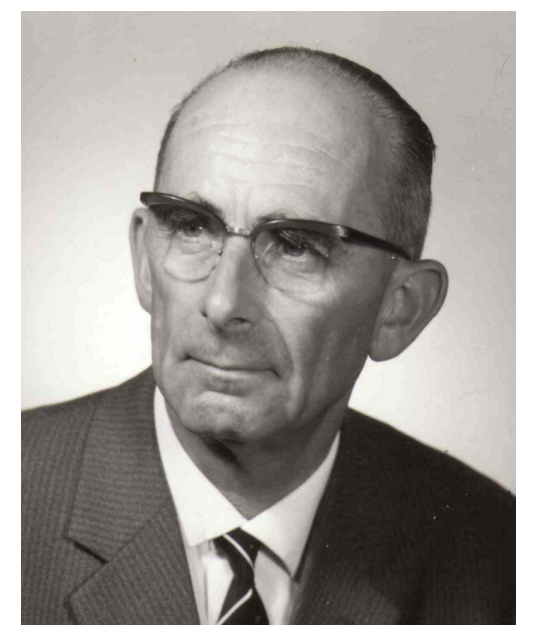

Figure 8. Rolf Wideröe (1902 - 1996). Courtesy of Pedro Waloschek.

Melvin Month was instrumental in creating the APS topical group on beam physics, which later became the APS Division of the Physics of Beams.

If one looks beyond the United States one sees institutions training accelerator scientists located in most of the advanced nations of the world. One notes institutions Aarhus University, Beijing University, the University of Bonn, Cambridge University, the ETH, Hiroshima University, The University of Kyoto, Moscow University, the University of Naples, Novosibirsk University, Oxford, the University of Pohang the University of Rome, the University of Paris, the Technical University of Berlin, Tel Aviv University, Tokyo University, Uppsala University, and very many other places.

The reader will recognize those institutions that were more active in the past than now, but he/she will also note, with satisfaction, the significant number of institutions that are currently very active in accelerator science and the training of accelerator scientists. Activity often comes down to a single person. There are large centers where there is more than one person, but that tends to be the exception. It is frequently the case that an institution offers instruction in accelerator and beam physics simply because one professor is active in the field and when he/she leaves that institution it sometimes takes many years before another person with similar interests is hired or the institution decides to put its emphasis in another direction. Conversely, institutions not previously teaching beam physics, with one hire, became active centers. We have seen that many times over and, most importantly, in developing universities, and in developing countries.

\section{The Laboratories}

The first accelerators were, most naturally, built in laboratories designed and operated for other kinds of physics. One thinks of the work of Cockcroft and Walton in the Cavendish Laboratory (Fig. 9), of Lawrence in Le Conte Hall on the Berkeley Campus, of Gregory Breit, Odd Dahl and Merle Tuve in the Department of Terrestrial Magnetism of the Carnegie Institute, and of Van de Graaff in the Palmer Physics Laboratory in Princeton. 


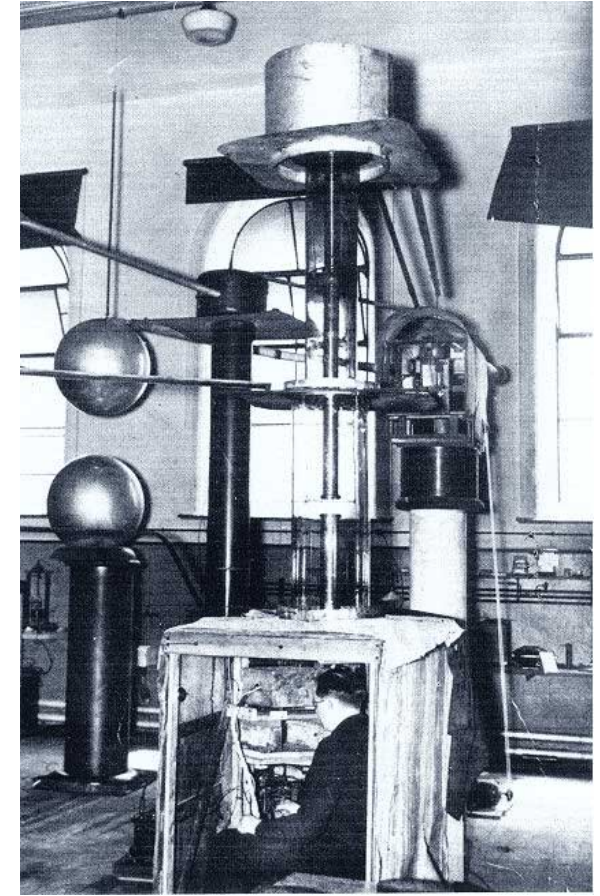

Figure 9. The original Cockcroft-Walton installation at the Cavendish laboratory. Walton is sitting in the cubicle. Courtesy of University of Cambridge, Cavendish Laboratory.

Soon that was to change. Van de Graaff moved to MIT and set up his large machines in an old aircraft hanger at the Round Hill Experimental Station. Lawrence moved to a special building on the Berkeley Campus, and so started, in 1931, the Radiation Laboratory (now LBNL). This growth was not without pains. Birge, then Chair of the UC Berkeley physics department remarked, "Berkeley has become less a university with a cyclotron than a cyclotron with a university attached." In 1940 the Rad Lab was moved to its present 200-acre location high in the Berkeley hills with a spectacular view of the San Francisco Bay Area (Fig. 10).

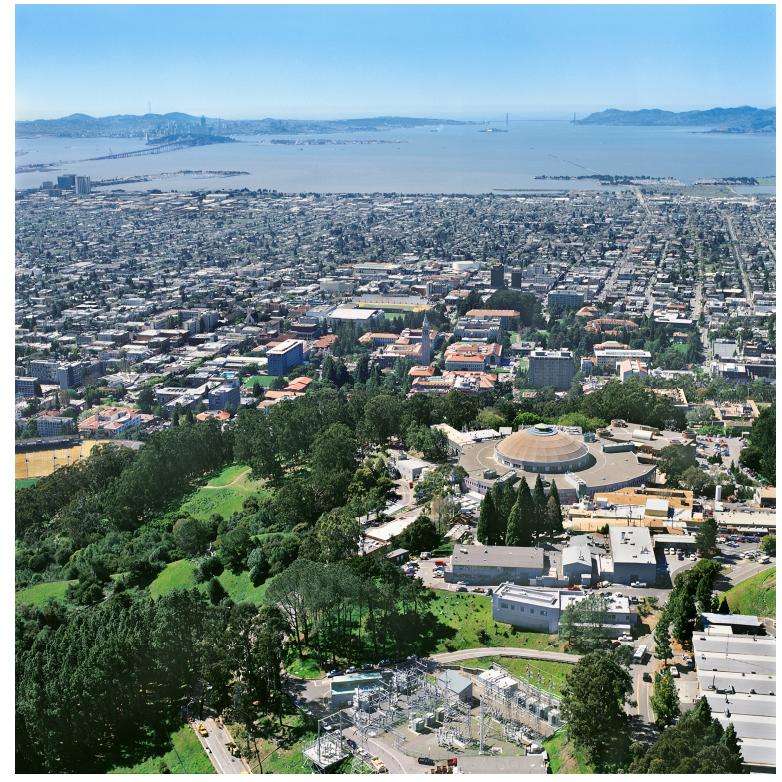

Figure 10. Lawrence Berkeley National Laboratory overlooking the San Francisco Bay Area. Courtesy of Lawrence Berkeley National Laboratory.

A number of universities, in many locations, were constructing in the 30's, cyclotrons and electrostatic machines and even betatrons. Often these machines required a special building, but were under the auspices of local physics departments. After WWII, driven by nuclear and then particle physics, a number of large specialized laboratories were developed. In the United States a number of these large laboratories had been developed during the war, and they were used to house accelerators and do experiments with them. The first laboratory devoted to accelerator science was Brookhaven where, first the Cosmotron was constructed, and then the AGS. At the same time the Rad Lab commissioned the 184-inch cyclotron, and then linacs, synchrotrons and the Bevatron. At the same time SLAC, building upon the initial Nobel Prize winning work at HEPL was initiated, but with some pains that closely remind one of Birge's remark. A series of electron synchrotrons was constructed at 
Cornell University, including the first synchrotron to incorporate strong focusing.

Major laboratories were also developed in the Soviet Union. Some, like the JINR at Dubna, was especially established for the construction and utilization of accelerators; others, such as Kurchatov, a laboratory built for another purpose was converted into an accelerator facility. One of the most important - and now the most important - was CERN. The creation of CERN was an effort to do what no European nation could do alone, namely build a large synchrotron (the PS). It was also important politically, as well as scientifically, as it was the first time that the former enemies of WWII worked together on a joint project. The formation of CERN had the support and help of the UN, just as the UN today is helping in the current activity to bring a Third Generation synchrotron radiation facility, SESAME, to the Middle East where both Israel and Arab nations are participating.

Soon, many other accelerator laboratories were created: Frascati (Italy), DESY (Germany), GSI (Germany), Serpukhov (Soviet Union), KEK (Japan), IHEP (China), INP (Soviet Union), SIN/PSI (Switzerland), Calcutta (India), RIKEN (Japan), TRIUMF (Canada), GANIL (France), RAL (England), Fermilab, JLab and Bates (United States). There are now 70 or so synchrotron laboratories in very many countries. Simultaneously increased accelerator construction activity occurred at the already mentioned laboratories, such as the ZGS and the superconducting ion linac at Argonne, RHIC at Brookhaven, LAMPF at Los Alamos, and the SNS at Oak Ridge.

Although the primary purpose of these laboratories was science, they actually served many purposes. In some cases, such as with CERN and SESAME, there is a positive political benefit to having rival countries work together. In some cases there was international rivalry, such as between Berkeley and Dubna over the discovery of transuranic elements, or the competition between Argonne and Dubna to build the highest energy proton accelerator (the ZGS and the Synchro-phasotron). Sometimes it was simply national prestige, and sometimes it was an effort to keep scientists at home and to stimulate and encourage young scientists to stay and work in their home countries. National prestige is important, for it has proved effective in getting countries, which at first had few or no accelerators, to the very forefront of our science.

\section{Communication and Interaction}

When we trace the development of communication and interaction between accelerator physicists, we find that it is really no different than the development of communication between all kinds of physicists (and, even, amongst the general population). Nevertheless, it is useful - so as to give us perspective - to look back just a bit.

Before WWII, although there were letters, and even visits, the primary method of communication - and, of course, interaction was at a conference, often an APS meeting. Ernest O. Lawrence, for example, would travel east once a year and it wasn't easy, as he would travel by train each way. In 1933 he was the only American to attend the Solvay Conference in Brussels. That required, both ways, a long train trip and an ocean voyage. And all this to report, unfortunately incorrectly, that the break-up of deuterium would be a power source.

Turning to the present, there is very extensive communication and interaction. Besides snail-mail and the telephone there is email, which is very widely used. (It is proper to note, if only parenthetically, that the World Wide Web, perhaps the most important development in communication and interaction in the late $20^{\text {th }}$ century, came from CERN.) There are international general conferences, PAC, EPAC, and APAC, operating on a rotating basis between regions so there is one conference each year. In addition, there are national conferences in the US (PAC in alternate years) and in Russia. There are specialized conferences such as COOL, CYCLOTRONS, DIPAC, FEL, ICALEPCS, 
ICAP, ICFA ABDW, and LINAC. Proceedings of these, as well as the general conferences are available on the Joint Accelerator Conferences Website (JACoW). In fact, JACoW has many Proceedings from earlier years, now scanned and available on their site. The American Physical Society DPB (Division of Physics of Beams) organizes accelerator physics sessions at APS meetings, and, with other APS Divisions, jointly sponsors sessions in which the scientists using accelerator beams report their results. DPB is a joint sponsor with IEEE of the PACs.

Of course, there are the many brief visits of accelerator scientists (now easy by plane) as well as longer-term visits. There are few accelerator scientists who have not spent some time at another institution, thus learning different approaches and making long-term contacts and friendships.

Finally, one notes the USPAS, the US Particle Accelerator School, started by Melvin Month, and now a solid institution. The US Particle Accelerator School provides educational programs in the field of beams and their associated accelerator technologies not otherwise available to the community, conducts graduate and undergraduate level courses at U.S. universities, holding two such programs per year, one in June and one in January. Students are welcomed from all corners of the world, from universities, laboratories, private companies, government and the military. Some students have been in the field for many years and are interested in a "refresher" course, while others are full-time students looking for additional classes to add to their education. Qualified teachers are chosen from national laboratories, universities and private industry. To carry out its educational mission, the USPAS develops programs of courses suitable for universities. Major universities, in partnership with the national laboratories, underwrite the offerings and provide the necessary quality control. Through this administrative framework, universities across the nation can offer highquality advanced technology courses. Similar schools are also held in Europe, and now in Asia. They have had an important role in developing expertise amongst scientists in the accelerator community.

\section{Part C. The Future}

It can be dangerous to try to predict the future. But, perhaps, it is not so dangerous in accelerator science, for the subject is rather mature and, at least for major machines, the time scale is long. Some would add "too long." For example the Next Linear Collider was first seriously studied in 1984 and will be initiated - at best - in the middle of the next decade, some 30 years later.

The long time scale for conceiving, designing, obtaining funding, choosing the site, constructing and commissioning a new big machine has a very large negative impact on careers and the training of students not to mention its effect upon the science. Too many of our colleagues are spending their whole scientifically productive lives making proposals and doing calculations on a machine that they may never see or even actually construct.

So, throwing caution to the wind, let us try to look into the future.

\section{Drivers}

Clearly, all of the "drivers" mentioned in Part A will continue to drive accelerator science for the next decades. Nuclear physicists, as already mentioned, will want to have electronion collisions and new accelerators built where they can study radioactive ion collisions.

High-energy physics will neither end nor can it manage without accelerators. Of course they desire to discover the Higgs, see whether super symmetry is a correct description of nature, solve the hierarchy problem, etc. and, perhaps most importantly, contribute to our understanding of dark matter and dark energy. Improvements to the LHC are in our future. Also, surely, we can look forward to the need for some smaller machines designed to study phi mesons, tau leptons, and various rare 
decays. And, of course, some day there will be a very high-energy electron linear collider: the International Linear Collider (ILC).

Just what form the ILC will take is uncertain. The decision has been made to use the low frequency superconducting $\mathrm{rf}$ (TESLA) technology. But funding is clearly coming much more slowly than the community expected. Since international agreements must also be made before construction can begin, it seems unlikely that we will have an ILC before the 2020 decade. In this situation, it is not obvious that a machine limited to low energy, as is the case for TESLA approach, is what the high-energy physics community will desire. If the ILC had been built rapidly and then followed with something else, like the TwoBeam Accelerator (TBA) approach of CLIC or a klystron powered X-Band device, it would be a different story. But if there will be only one ILC for as far into the future as one can envision (perhaps until 2050, or beyond) the technology choice may be very different. In any case, we must wait for LHC data. It will not be a surprise if the decision as to what form the ILC will take is revisited. But, in any case, sooner or later, there will be plenty of need for accelerator expertise.

In addition to the above there is a compelling physics case to be made for the creation of super neutrino beams and, some day, a neutrino factory based upon the capture, cooling, and acceleration of muons, whose subsequent decay produces neutrinos. Also, of course, in our future may be a muon collider.

Condensed matter physics, chemistry and biology have uses for synchrotron radiation that will stimulate and justify the construction of ever-more Third Generation facilities. One was just completed in Australia, and surely that model will be followed in many other countries. The desire to obtain brief, coherent, high repetition rate, X-ray pulses will drive the development of Fourth Generation light sources. Perhaps more than any other need, the development of Fourth Generation facilities will be the "driver" of future accelerator science. It seems clear that one can look forward to a great deal of accelerator and beam science, including many advanced technology developments in creating the Fourth Generation Facilities desired by our scientific colleagues.

Also, of course, there will be complementary neutron spallation facilities. In the US and Japan there are already major facilities, but surely similar devices will be built in other places. China has already begun and Europe has had plans for a rather long time to build a neutron spallation source.

National defense, cancer therapy and industry can be counted upon to have needs as far into the future as one can imagine. Surely, these applications alone, will keep the field healthy and vibrant.

\section{New Accelerator Science}

Driven by the physics and application needs, but almost independent of them has been, and will continue to be, the development of accelerator science. In the past, it has been realized by the funding agencies that a certain amount of money should be spent in such an undirected manner. We can hope - and should do what we can to ensure it - that this will continue into the future.

Looking back, we can see so many wonderful advances, most of which, at least at first, came out of undirected research. One thinks of phase focusing, strong focusing, lattice design methods, non-linear dynamics studies, electron cooling, stochastic cooling, laser cooling, RFQ's, FFAG, colliding beams, low beta insertions, instability theory, numerical computation of magnets and rf cavities, superconducting magnets and superconducting rf, vacuum technology, linac design, injection and extraction kickers, FELs, and on and on.

And, it would be wrong to think we are at the end of making any further discoveries and developments. Besides the incremental improvement of what we know, there will surely be developments we cannot even envision. One has only to attend one of the specialty conferences, say a COOL Conference 
or a Neutrino Factory and Muon Collider Collaboration meeting or an FEL Conference, to witness the excitement and innovation of accelerator science.

As we mentioned above, the CLIC scheme is a possibility for the ILC and, most people agree, a very serious possibility for the linear collider beyond the ILC. At CERN a significant effort of study has been under way and will continue into the future. There have been major proof-of-principle and prototyping of parts of the Two-Beam Accelerator approach. We can be confident of a continuation of this effort.

Motivated by the possibility of making a neutrino factory and, also, a muon collider, a great deal of effort has gone into the study of accelerating a muon beam. Since muons are produced in nuclear decay of pions the beam emittance is large and cooling is necessary. Only ionization cooling is fast enough compared to the muon decay time of a few microseconds (in the muon rest frame), and much thought has gone into the development of configurations that will give the necessary 3D damping. An experiment to demonstrate ionization cooling (MICE) is underway at RAL. The acceleration of muons could be done in a recirculating linac or, possibly, in an FFAG structure. An experiment to study NonScaling FFAG acceleration is now underway (EMMA) at Daresbury. Also, this activity has stimulated the experimental realization of optical stochastic cooling, which is, of course, interesting in its own right.

It is possible to drive a sub-critical reactor with an incoming neutron beam. That would increase the safety of a reactor (since the driving beam can simply be switched off). Experimental demonstration of this idea is being done at KURI (part of Kyoto University). Of course there are many other possible sources of accidents, like a fire or sabotage that this does not address. Perhaps more interesting, is the use of an accelerator to breed thorium into a fuel. And, finally, accelerator beams can be used to burn up the actinides and thus reduce the time that nuclear waste must be isolated from the environment from hundreds of thousands of years to hundreds of years. Designs that do all three things have been studied in Europe, Japan and in the US at LANL. Burning thorium is of interest to India for they don't have uranium but do have thorium. Burning actinides is of considerable interest and is part of the Global Nuclear Energy Partnership (GNEP). However, GNEP envisions advanced breeder reactors rather than accelerators for this purpose. The situation is in flux and it isn't clear whether accelerators will ever be used for these purposes, but maybe they will be.

A second energy production use of accelerators is in fusion. Effort on heavy ion inertial fusion has been going on for the last 30 years. The Europeans at GSI have followed an approach with rf accelerators, while the Americans (mostly at LBNL, but with Princeton and LLNL also involved) have studied an induction accelerator approach. Both programs have suffered by not having sufficient support. It is not clear that heavy ion inertial fusion will be the answer to mankind's need for energy, nor is it even clear that it will play a significant role. But the energy problem is so pressing that it is disappointing, almost unbelievable, that even the modest financial support needed to significantly increase the rate of progress in this work, is not forthcoming.

In the last few years interest has grown in high energy density physics. The applications are very broad and range from astrophysics to national defense. Often the method to obtain high energy density is with accelerators. Of course lasers - such as in NIF - also provide an excellent source. To the dismay of many the heavy ion fusion program has been redirected to high energy density studies. Yes, the two are related, but pursuit of high energy density, which is an important subject, should not have been used to slow down the fusion effort. The world needs both.

We want to note the very interesting beam physics that is being driven by some of the new projects like FAIR at GSI and ELENA at 
CERN. The cooling of stored beams is an exciting science and relevant to many applications (such as has already been seen at the Tevatron and at RHIC). The production of cold beams is of much interest for FELs and also, for example, making the very cold, and almost stopped, antiproton beams. These are used for the study of anti hydrogen, a challenge undertaken at CERN and soon to be undertaken at FAIR.

Finally, we note, with enthusiasm, the very extensive effort, and considerable success, in the development of wholly new methods of acceleration. The work on laser and laser/plasma acceleration has been going on since 1978, with a great deal of progress, but no practical accelerator yet achieved. The development of accelerating plasma channels, so that a laser beam remains confined beyond the Rayleigh Length, and the subsequent acceleration of electrons by a powerful laser to high energy (more than a GeV) and with a very small energy spread has already been achieved. In another approach, the wake field from an intense, and longitudinally compact, bunch has been used to accelerate a second group of particles. In an experiment at SLAC a beam of $45 \mathrm{GeV}$ was used to more than double the energy of another beam of electrons.

The present laser driven accelerator experiments employ rather expensive and inefficient lasers. But laser technology has, ever since the invention of the laser, made continual and significant progress. It is reasonable to assume that further advances will continue to be made and that efficient and inexpensive lasers, suitable for laser/plasma acceleration, are in our future. If that is to be the case, then laser/plasma accelerators will become available for various applications, but probably not for high-energy physics, at least not for very many years.

\section{Final Words}

Having gotten this far, the reader will have seen that we have tried to answer the questions listed in the Introduction. We have delineated the many very powerful "drivers" of accelerator science and have commented upon the many interesting and innovative aspects of accelerator science. It is clear that the field of accelerator and beam physics will remain vibrant and interesting for many more years. Given the necessary long-term investment in education and research and development, it is important to see far enough into the future to be confident that young scientists and students specializing in accelerator physics are not being "led down a primrose path." We are sure it is quite the contrary, and hopefully this short article has convinced the reader of the bright future of our science.

Thus it appears proper to attract and train young people in this field, all the while being confident that they will have both future employment and an exciting scientific career. In short, that they will have as much fun as we have had.

\section{Acknowledgments}

We thank Ka-Ngo Leung for assistance with Chapter 7. 